\title{
Observations on interpolation by total degree polynomials in two variables
}

\author{
Jesús Carnicer • Tomas Sauer
}

\begin{abstract}
In contrast to the univariate case, interpolation with polynomials of a given maximal total degree is not always possible even if the number of interpolation points and the space dimension coincide. Due to that, numerous constructions for interpolation sets have been devised, the most popular ones being based on intersections of lines. In this paper, we study algebraic properties of some such interpolation configurations, namely the approaches by Radon-Berzolari and Chung-Yao. By means of proper H-bases for the vanishing ideal of the configuration, we derive properties of the matrix of first syzygies of this ideal which allow us to draw conclusions on the geometry of the point configuration.
\end{abstract}

Keywords Polynomial interpolation · syzygies · H-bases

Mathematics Subject Classification (2000) 41A05 - 13P10

\section{Introduction}

Interpolation of data, especially by polynomials, is a classical issue, not only in one variable but also in several variables, cf. $[14,19]$. While in one variable the interpolation polynomial can be easily expressed in a closed form, this is not the case in two and more variables where the geometry becomes significantly

J. Carnicer was partially supported by MTM2015-65433-P (MINECO/FEDER) Spanish Research Grant, by Gobierno de Aragón and European Social Fund.

J. Carnicer

Departamento de Matematica Aplicada/IUMA, Universidad de Zaragoza, Pedro Cerbuna

12, 50009 Zaragoza, Spain

E-mail: carnicer@unizar.es

T. Sauer

Universität Passau, Lehrstuhl für Mathematik mit Schwerpunkt Digitale Bildverarbeitung \& FORWISS, Innstr. 43, D-94032 Passau, Germany 
more intricate, especially due to the fact that there are no multivariate Haar spaces, see [16, Chapter 2, Section 4].

To overcome some of these problems, techniques were developed to construct point sets that allow for unique interpolation from a given subspace of polynomials, typically the vector space of all polynomials of total degree not greater than a given number. The Radon-Berzolari construction $[1,17]$ generates a set of interpolation points by decomposing a bivariate problem of degree, say $n$, into two simpler subproblems, one being univariate and one of degree $n-1$ : the construction extends a set of interpolation points for degree $n-1$ by choosing $n+1$ additional points on a line. As long as this line does not contain any of the low degree interpolation points, this gives a valid set of interpolation points of degree $n$.

Later, Chung and Yao [10] presented a geometric characterization of certain interpolation sets that extend properties of the univariate case to several variables. In particular, they defined a class of interpolation sets which are nowadays known as GC configurations [5] or GC sets [4,9]. Gasca and Maeztu [13] conjectured that any bivariate GC set is the result of a Berzolari-Radon construction, that is, any such set possesses a maximal line which contains $n+1$ of the interpolation points. This conjecture, based on the simple observation in the cases $n=1,2$, has so far only been proven for degree up to 5 , see [15].

Recently, Hal Schenck pointed out the striking connections between interpolation sets and the generating matrix for the first syzygy module of the associated zero dimensional radical ideal. This approach which is based on sophisticated concepts from Algebraic Geometry, see [11], can be found in [12]. One main point there is that it is possible to characterize the existence of a maximal line in an interpolation set by looking at the syzygy matrix of the respective ideal. The purpose of this paper is to give an elementary and direct approach to these ideas in which the Berzolari-Radon construction plays a significant role.

\section{Interpolation of total degree and ideals}

Let $\Pi=\mathbb{R}[x], x=\left(x_{1}, x_{2}\right)$, be the set of bivariate polynomials and $\Pi_{n}$ be the set of bivariate polynomials of total degree less than or equal to $n$ whose dimension is $\operatorname{dim} \Pi_{n}=(n+1)(n+2) / 2$. Given a set $Y \subseteq \mathbb{R}^{2}$, the evaluation map is defined as

$$
p \in \Pi \mapsto p(Y):=(p(y))_{y \in Y} \in \mathbb{R}^{Y} .
$$

The kernel of the evaluation map is the ideal

$$
I(Y):=\{p \in \Pi: p(Y)=0\}
$$

of all bivariate polynomials vanishing at the set $Y$.

Given a finite set of nodes $Y \subset \mathbb{R}^{2}$ and $f \in \mathbb{R}^{Y}$, we can formulate the Lagrange interpolation problem on $Y$ in $\Pi_{n}$ : find $p \in \Pi_{n}$ such that $p(Y)=f$. 
Definition $1 \mathrm{~A}$ set $Y \subset \mathbb{R}^{2}$ is $\Pi_{n}$-independent if the Lagrange interpolation problem on $Y$ has a solution in $\Pi_{n}$, maybe not unique. A set $Y \subset \mathbb{R}^{2}$ is $\Pi_{n}$-poised if the interpolation problem on $Y$ in $\Pi_{n}$ is unisolvent, that is, the interpolation problem on $Y$ has always a unique solution in $\Pi_{n}$.

The evaluation map is a surjective linear map for any finite $Y$ since for each $f \in \mathbb{R}^{Y}$ the polynomial

$$
p(x)=\sum_{y \in Y} f(y) \prod_{t \in Y \backslash\{y\}} \frac{\left(x_{1}-t_{1}\right)^{2}+\left(x_{2}-t_{2}\right)^{2}}{\left(y_{1}-t_{1}\right)^{2}+\left(y_{2}-t_{2}\right)^{2}}
$$

satisfies $p(Y)=f$. Hence, $\mathbb{R}^{Y} \cong \Pi / I(Y)$.

The set $Y$ is $\Pi_{n}$-poised if and only if the restriction of the evaluation map to $\Pi_{n}$ is bijective. So, if $Y$ is $\Pi_{n}$-poised then $\operatorname{dim} \Pi_{n}=\operatorname{dim} \mathbb{R}^{Y}=\# Y$. Uniqueness of the solution of the Lagrange interpolation problem implies that $\Pi_{n} \cap I(Y)=0$ and existence of a solution implies that $\Pi=\Pi_{n}+I(Y)$. Therefore $Y$ is $\Pi_{n}$-poised if and only if $\Pi_{n} \oplus I(Y)=\Pi$.

If $Y$ is a $\Pi_{n}$-poised set, then for each $y \in Y$ there exists a unique fundamental polynomial, also called Lagrange polynomial, $\ell_{y, Y}$ in $\Pi_{n}$ such that

$$
\ell_{y, Y}\left(y^{\prime}\right)=\delta_{y y^{\prime}}, \quad y^{\prime} \in Y,
$$

where $\delta_{y y^{\prime}}$ is Kronecker's symbol. For a $\Pi_{n}$-poised set $Y$ the Lagrange interpolation operator $L_{Y}$ associates to each function from $\mathbb{R}^{2}$ to $\mathbb{R}$ its polynomial interpolant in $\Pi_{n}$. Considered as an operator from $\Pi$ to $\Pi$, the Lagrange interpolation operator is a projector that can be expressed in terms of the fundamental polynomials by means of the Lagrange formula

$$
L_{Y}[f]=\sum_{y \in Y} f(y) \ell_{y, Y} .
$$

The error operator

$$
E_{Y}[f]:=f-L_{Y}[f]
$$

is another linear projector whose image is the ideal $I(Y)$, and the two projectors are complementary. Because of that the Lagrange interpolation operator is called an ideal projector, that is, a projector whose kernel is an ideal [3].

From the definition it follows that $Y$ is a $\Pi_{n}$-independent set if and only if for each $y \in Y$ there exists a fundamental polynomial (maybe not unique) in $\Pi_{n}$ vanishing at $Y \backslash\{y\}$ and with value 1 at $y$. Observe that a subset of a $\Pi_{n}$-independent set is also $\Pi_{n}$-independent. For each $\Pi_{n}$-independent set we have that $\# Y \leq \operatorname{dim} \Pi_{n}$ and, if equality holds, then $Y$ is $\Pi_{n}$-poised.

Definition 2 A subset $H$ of an ideal $I$ is called an $H$-basis for $I$ if any $f \in I$ can be written as

$$
f=\sum_{h \in H} g_{h} h, \quad g_{h} \in \Pi_{\operatorname{deg} f-\operatorname{deg} h}, \quad h \in H .
$$


Lemma 1 Let $I$ be an ideal of $\Pi$ such that $\Pi_{n} \cap I=0$ and $\operatorname{dim}\left(\Pi_{n+1} \cap I\right)=$ $n+2$. Then $h_{0}, \ldots, h_{n+1}$ is a basis of $\Pi_{n+1} \cap I$ if and only if it is an $H$-basis of $I$. Moreover, $\Pi=\Pi_{n} \oplus I$.

Proof Let $h_{0}, \ldots, h_{n+1}$ be a basis of $\Pi_{n+1} \cap I$. Since $\Pi_{n} \cap I=\{0\}$, we have that $\Pi_{n+1}=\Pi_{n} \oplus\left(\Pi_{n+1} \cap I\right)$ and for each $\alpha$ with $|\alpha|=n+1$ there exists $g_{\alpha}$ in the vector space $\Pi_{n+1} \cap I$ such that the $g_{\alpha}(x)-x^{\alpha} \in \Pi_{n}$. Since $x^{\alpha},|\alpha|=n+1$, are linearly independent, the polynomials $g_{\alpha}(x),|\alpha|=n+1$, are also linearly independent and, since $\operatorname{dim}\left(\Pi_{n+1} \cap I\right)=n+2$, they are a basis of $\Pi_{n+1} \cap I$. Now take any polynomial $p \in \Pi_{m}, m \geq n+1$. The homogeneous leading form of $p$ can be expressed as $\sum_{|\alpha|=n+1} c_{\alpha}(x) x^{\alpha}$ with $c_{\alpha}(x) \in \Pi_{m-n-1}$. Then

$$
p-\sum_{|\alpha|=n+1} c_{\alpha} g_{\alpha} \in \Pi_{m-1} .
$$

An inductive argument shows that this reduction process allows us find polynomials $b_{\alpha} \in \Pi_{m-n-1},|\alpha|=n+1$, such that

$$
r:=p-\sum_{|\alpha|=n+1} b_{\alpha} g_{\alpha} \in \Pi_{n} .
$$

Hence it follows that $\Pi=\Pi_{n} \oplus I$. If $p \in I$ then $r \in \Pi_{n} \cap I=0$, hence $p$ can be expressed as a linear combination of the polynomials $g_{\alpha}$, that is, with polynomial coefficients $b_{\alpha} \in \Pi_{m-n-1},|\alpha|=n+1$. So, $\left(g_{\alpha}:|\alpha|=n+1\right)$ is an $H$-basis. Since each $g_{\alpha} \in \Pi_{n+1} \cap I$ can be expressed as a linear combination of the basis $\left(h_{0}, \ldots, h_{n}\right)$ with constant coefficients and vice versa, it follows that $\left(h_{0}, \ldots, h_{n}\right)$ is also an H-basis.

Conversely, if $h_{0}, \ldots, h_{n+1}$ is an H-basis and $p \in \Pi_{n+1} \cap I$, then $p$ can be represented with respect to the $h_{j}, j=0, \ldots, n+1$, and the polynomial coefficients must have degree 0 . Hence $\operatorname{span}\left\{h_{0}, \ldots, h_{n+1}\right\}=\Pi_{n+1} \cap I$ and, since $\operatorname{dim}\left(\Pi_{n+1} \cap I\right)=n+2$, it follows that $h_{0}, \ldots, h_{n+1}$ is a basis of the vector space $\Pi_{n+1} \cap I$.

The statement of the preceding lemma can be rephrased as follows: for any ideal $I$ of $\Pi$ the conditions $\Pi_{n} \cap I=\{0\}$ and $\operatorname{dim}\left(\Pi_{n+1} \cap I\right)=n+2$ are equivalent to $\Pi=\Pi_{n} \oplus I$.

Corollary 1 Let $I$ be an ideal of $\Pi$ such that $\Pi_{n} \cap I=\{0\}$ and $\operatorname{dim}\left(\Pi_{n+1} \cap\right.$ $I)=n+2$ and let $P$ be a finite spanning subset of $\Pi_{n+1} \cap I$. Then $P$ is an $H$-basis of $I$.

\section{Some remarks on the Berzolari-Radon construction}

For each set $P$ of bivariate polynomials, the associated algebraic variety is defined as $V(P):=\left\{x \in \mathbb{R}^{2}: p(x)=0, p \in P\right\}$. If $P$ consists of a single polynomial $p$, we shall denote by $P$ the algebraic curve with equation $p(x)=0$ 
for the sake of brevity, instead of using the notation $V(P)$. A line is the set of zeros of a bivariate polynomial of first degree.

Berzolari [1] and Radon [17] proposed the construction of a $\Pi_{n+1}$-poised set $Y_{n+1}$ starting from a $\Pi_{n}$-poised set $Y_{n}$ by adding $n+2$ nodes lying on a line $W$ that does not contain any node in $Y_{n}$. In Fact (3) of [4], we can find a proof of this result and a relation between the fundamental polynomials of both sets. We provide a restatement of these results, providing explicit relations between the corresponding fundamental polynomials that will be used afterwards.

In the sequel we will use the convenient abbreviation $T:=Y_{n+1} \cap W=$ $Y_{n+1} \backslash Y_{n}$.

Theorem 1 Let $Y_{n+1}$ be an $\Pi_{n+1}$-poised set and $W$ be a line such that \# $(W \cap$ $\left.Y_{n+1}\right)=n+2$. Then the set $Y_{n}:=Y_{n+1} \backslash W$ is $\Pi_{n}$-poised.

Conversely, if $Y_{n}$ is a $\Pi_{n}$-poised set with $Y_{n} \cap W=\emptyset$ and $Y_{n+1}$ is obtained by adding to $Y_{n} n+2$ distinct nodes on the line $W$, then $Y_{n+1}$ is $\Pi_{n}$-poised. Moreover,

$$
\ell_{y, Y_{n+1}}(x)=\frac{w(x)}{w(y)} \ell_{y, Y_{n}}(x), \quad y \in Y_{n},
$$

and, for $t \in T$,

$$
\ell_{t, Y_{n+1}}(x)=\prod_{s \in T \backslash\{t\}} \frac{m(x)-m(s)}{m(t)-m(s)}-w(x) \sum_{y \in Y_{n}} \frac{\ell_{y, Y_{n}}(x)}{w(y)} \prod_{s \in T \backslash\{t\}} \frac{m(y)-m(s)}{m(t)-m(s)},
$$

where $m$ is an arbitrary polynomial of first degree such that $1, w, m$ form a basis of $\Pi_{1}$ and $T:=Y_{n+1} \cap W$.

Proof For each $t \in T$, we define the polynomial

$$
d_{t}(x):=\prod_{s \in T \backslash\{t\}}(m(x)-m(s)) .
$$

Since $d_{t}(t) \neq 0$, we can find a fundamental polynomial of the form

$$
q_{t}(x):=\frac{d_{t}(x)}{d_{t}(t)}=\prod_{s \in T \backslash\{t\}} \frac{m(x)-m(s)}{m(t)-m(s)} .
$$

for each $t \in T$. So, $T$ is a $\Pi_{n+1}$-independent set. The restriction of the evaluation map $p \mapsto p(T)$ to $\Pi_{n+1}$ is surjective because $T$ is $\Pi_{n+1}$-independent and its kernel $\left\{p \in \Pi_{n+1}: p(T)=0\right\}$ has dimension $\operatorname{dim} \Pi_{n+1}-(n+2)=\operatorname{dim} \Pi_{n}$. Since $w \Pi_{n}:=\left\{w p: p \in \Pi_{n}\right\}$ is contained in its kernel and since both spaces have the same dimension they must coincide.

Assume that $Y_{n+1}$ is $\Pi_{n+1}$-poised and let $y \in Y_{n}:=Y_{n+1} \backslash W$. We have that $\ell_{y, Y_{n+1}}(T)=0$ which implies $\ell_{y, Y_{n+1}} \in w \Pi_{n}$. Therefore, $w$ is a factor of $\ell_{y, Y_{n+1}}$ and $w(y) \ell_{y, Y_{n+1}} / w$ is a fundamental polynomial in $\Pi_{n}$ of $y$ for the Lagrange interpolation problem in $Y_{n}$. Hence $Y_{n}$ is a $\Pi_{n}$-independent set and since $\# Y_{n}=\# Y_{n+1}-(n+2)=\operatorname{dim} \Pi_{n}$, it follows that $Y_{n}$ is $\Pi_{n}$-poised, proving the first statement. 
Conversely, if $Y_{n}$ is $\Pi_{n}$-poised, then $w \ell_{y, Y_{n}} / w(y)$ is a fundamental polynomial in $\Pi_{n}$ of $y$ for the Lagrange problem in $Y_{n+1}$ for each $y \in Y_{n}$. Now take $t \in T$. Since $w$ does not vanish on the set $Y_{n}$ and $Y_{n}$ is $\Pi_{n}$-poised, we can define a polynomial interpolating $q_{t} / w$ on $Y_{n}$

$$
L_{Y_{n}}\left[q_{t} / w\right]=\sum_{y \in Y_{n}} \frac{q_{t}(y)}{w(y)} \ell_{y, Y_{n}}
$$

and deduce that $q_{t}(x)-w(x) L_{Y_{n}}\left[q_{t} / w\right](x)$ is a fundamental polynomial for $t$ in $Y_{n+1}$. Hence $Y_{n+1}$ is $\Pi_{n+1}$-independent and $\Pi_{n+1}$-poised since $\# Y_{n+1}=$ $\# Y_{n}+(n+1)=\operatorname{dim} \Pi_{n+1}$. Finally, (1) follows from

$\ell_{t, Y_{n+1}}(x)=q_{t}(x)-w(x) L_{Y_{n}}\left[q_{t} / w\right](x)=\frac{d_{t}(x)}{d_{t}(t)}-\frac{w(x)}{d_{t}(t)} \sum_{y \in Y} d_{t}(y) \frac{\ell_{y, Y}(x)}{w(y)}, \quad t \in T$.

Theorem 2 Let $Y_{n}$ be $\Pi_{n}$-poised, and let $T$ be any set of $n+2$ distinct points such that $Y_{n+1}=Y_{n} \cup T$ is a $\Pi_{n+1}$-poised set. Then the $n+2$ functions

$$
h_{t}:=\ell_{t, Y_{n+1}} \in \Pi_{n+1} \cap I\left(Y_{n}\right), \quad t \in T,
$$

are an H-basis of the ideal $I\left(Y_{n}\right)$.

Proof Clearly $\ell_{t, Y_{n+1}} \in \Pi_{n+1}$ vanish on $Y_{n}$ and so $h_{t}=\ell_{t, Y_{n+1}} \in \Pi_{n+1} \cap I\left(Y_{n}\right)$, $t \in T$. Since these Lagrange fundamental polynomials are linearly independent, they form a basis of $\Pi_{n+1} \cap I\left(Y_{n}\right)$. From the fact that $Y_{n}$ is $\Pi_{n}$-poised, it follows that $\Pi=\Pi_{n} \oplus I\left(Y_{n}\right)$ and together with the linear independence of the Lagrange fundamental polynomials this yields $\operatorname{dim} \Pi_{n+1} \cap I\left(Y_{n}\right)=\operatorname{dim} \Pi_{n+1}-$ $\operatorname{dim} \Pi_{n}=n+2$. Now we conclude from Lemma 1 that $\left(h_{t}: t \in T\right)$ is an $\mathrm{H}$ basis for $I$.

Theorem 1 and Theorem 2 can be combined to obtain an H-basis of the ideal $I(Y)$ of any $\Pi_{n}$-poised set $Y$ formed by fundamental polynomials obtained from the Berzolari-Radon construction.

Theorem 3 Let $Y_{n}$ be a $\Pi_{n}$-poised set, and $T$ be any set of $n+2$ points lying on a line $W$ such that $W \cap Y_{n}=\emptyset$ and $Y_{n+1}:=Y_{n} \cup T$. Then the $n+2$ polynomials

$$
h_{t}:=\ell_{t, Y_{n+1}} \in \Pi_{n+1} \cap I\left(Y_{n}\right), \quad t \in T,
$$

are $\mathbb{R}[w]$-independent and form an H-basis of the ideal $I\left(Y_{n}\right)$.

Proof By Theorem 1, $Y_{n+1}$ is $\Pi_{n+1}$-poised. The polynomials $\ell_{t, Y_{n+1}} \in \Pi_{n+1}$, $t \in T$, vanish on $Y_{n}$. Let us show now that $h_{t}=\ell_{t, Y_{n+1}} \in \Pi_{n+1} \cap I\left(Y_{n}\right), t \in T$, are $\mathbb{R}[w]$-independent, that is, if

$$
\sum_{t \in T} c_{t}(w) h_{t}=0,
$$


for some univariate polynomials $c_{t}, t \in T$, then all these polynomials are zero: $c_{t}=0, t \in T$. If we denote by $m_{t}$ the degree of $c_{t}$, we can write

$$
\sum_{t \in T} \sum_{j=0}^{m_{t}} c_{t, j} w^{j} h_{t}=0
$$

After dividing the above equation by an appropriate power of $w$, we may assume that $\left(c_{t, 0}: t \in T\right) \neq 0$. Then

$$
\sum_{t \in T} c_{t, 0} h_{t}=-\sum_{t \in T} \sum_{j=1}^{m_{t}} c_{t, j} w^{j} h_{t}=w q
$$

for some $q \in \Pi_{n}$. Since $w$ does not vanish at any node of $Y_{n}$, we have that $q\left(Y_{n}\right)=0$, that is, $q \in \Pi_{n} \cap I\left(Y_{n}\right)$. But $\Pi_{n} \cap I\left(Y_{n}\right)=\{0\}$ because $Y_{n}$ is $\Pi_{n}$-poised. So $q=0$ and from the linear independence of the Lagrange fundamental polynomials we deduce that $c_{t, 0}=0$ for each $t \in T$. So, the $\mathbb{R}[w]$ independence follows. The H-basis property follows from Theorem 2.

Definition 3 A syzygy of $P:=\left(p_{0}, \ldots, p_{n+1}\right) \in \Pi^{n+2}$ is $\Sigma:=\left(\sigma_{0}, \ldots, \sigma_{n+1}\right) \in$ $\Pi^{n+2}$ such that

$$
\sum_{i=0}^{n+1} \sigma_{i} p_{i}=0
$$

The set of all syzygies for $P$ will be denoted by $S(P)$ and the set of all syzygies of certain maximal degree $m$ as $S_{m}(P)=S(P) \cap \Pi_{m}^{n+2}, m \in \mathbb{N}_{0}$.

Intuitively, syzygies describe ambiguities in representing a polynomial with respect to an ideal. Indeed,

$$
f=\sum_{i=0}^{n+1} f_{i} p_{i}=\sum_{i=0}^{n+1} f_{i}^{\prime} p_{i}
$$

holds if and only if $\left(f_{0}-f_{0}^{\prime}, \ldots, f_{n+1}-f_{n+1}^{\prime}\right) \in S(P)$. The set of $S(P)$ of syzygies of $P \in \Pi^{n+2}$ forms a $\Pi$-submodule of $\Pi^{n+2}$. If $P$ is the basis of an ideal, $S(P)$ provides important information on the ways of expressing a polynomial in the ideal in terms of the basis $P$.

We have seen that for a $\Pi_{n}$-poised set $Y_{n}$ we can obtain an H-basis $h_{t}(x):=$ $\ell_{t, Y_{n+1}}(x), t \in T$, of the ideal $I\left(Y_{n}\right)$ using some of the Lagrange fundamental polynomials with respect to a set $Y_{n+1}=Y_{n} \cup T$ where $T$ is a set of $n+2$ points on a line $W$ such that $W \cap Y_{n}=\emptyset$. We also recall that the function

$$
d_{t}(x)=\prod_{s \in T \backslash\{t\}}(m(x)-m(s)), \quad t \in Y_{n+1},
$$

satisfies

$$
d_{t}(t) \neq 0
$$


and can be used to express the Lagrange fundamental polynomials described in (1)

$$
h_{t}(x)=\ell_{t, Y_{n+1}}(x)=\frac{d_{t}(x)}{d_{t}(t)}-\frac{w(x)}{d_{t}(t)} \sum_{y \in Y_{n}} d_{t}(y) \frac{\ell_{y, Y_{n}}(x)}{w(y)}
$$

where $m$ is a linear polynomial such that $\operatorname{span}\{1, w, m\}=\Pi_{1}$.

First note that by the $\mathbb{R}[w]$-independence, any nontrivial syzygy cannot consist of polynomials in $\mathbb{R}[w]$ only and some coefficient should include the independent polynomial $m$. In order to find explicit syzygies, we begin with $t_{i}, t_{j} \in T:=\left\{t_{0}, t_{1}, \ldots, t_{n+1}\right\}$ and compute

$$
\begin{aligned}
& d_{t_{i}}\left(t_{i}\right)\left(m(x)-m\left(t_{i}\right)\right) h_{t_{i}}(x)-d_{t_{j}}\left(t_{j}\right)\left(m(x)-m\left(t_{j}\right)\right) h_{t_{j}}(x) \\
& =w(x) \sum_{y \in Y_{n}}\left(d_{t_{i}}(y)\left(m(x)-m\left(t_{i}\right)\right)-d_{t_{j}}(y)\left(m(x)-m\left(t_{j}\right)\right)\right) \frac{\ell_{y, Y_{n}}(x)}{w(y)} .
\end{aligned}
$$

The left hand side of the above equation (3) belongs to $I\left(Y_{n}\right)$ and $w$ does not vanish at any point of $Y_{n}$, hence

$$
\sum_{y \in Y_{n}}\left(d_{t_{i}}(y)\left(m(x)-m\left(t_{i}\right)\right)-d_{t_{j}}(y)\left(m(x)-m\left(t_{j}\right)\right)\right) \frac{\ell_{y, Y_{n}}(x)}{w(y)} .
$$

is a polynomial in $\Pi_{n+1} \cap I\left(Y_{n}\right)$. Since $\left\{h_{t}: t \in T\right\}$ is a basis of the vector space $\Pi_{n+1} \cap I\left(Y_{n}\right)$, it follows that

$$
\sum_{y \in Y_{n}}\left(d_{t_{i}}(y)\left(m(x)-m\left(t_{i}\right)\right)-d_{t_{j}}(y)\left(m(x)-m\left(t_{j}\right)\right)\right) \frac{\ell_{y, Y_{n}}(x)}{w(y)}=\sum_{s \in T} c_{s}^{t_{i}, t_{j}} h_{s}(x),
$$

for coefficients $c_{s}^{t_{i}, t_{j}} \in \mathbb{R}$. Since $h_{s}:=\ell_{s, Y_{n+1}}(x)$ are fundamental polynomials in $T$, we even have the explicit formula

$$
c_{s}^{t_{i}, t_{j}}=\sum_{y \in Y_{n}}\left(d_{t_{i}}(y)\left(m(s)-m\left(t_{i}\right)\right)-d_{t_{j}}(y)\left(m(s)-m\left(t_{j}\right)\right)\right) \frac{\ell_{y, Y_{n}}(s)}{w(y)} .
$$

In particular,

$$
\begin{aligned}
c_{t_{i}}^{t_{i}, t_{j}} & =-\left(m\left(t_{i}\right)-m\left(t_{j}\right)\right) \sum_{y \in Y_{n}} d_{t_{j}}(y) \frac{\ell_{y, Y_{n}}\left(t_{i}\right)}{w(y)}, \\
c_{t_{j}}^{t_{i}, t_{j}} & =\left(m\left(t_{j}\right)-m\left(t_{i}\right)\right) \sum_{y \in Y_{n}} d_{t_{i}}(y) \frac{\ell_{y, Y_{n}}\left(t_{j}\right)}{w(y)} .
\end{aligned}
$$

Then formula (3) can be written in the form

$d_{t_{i}}\left(t_{i}\right)\left(m(x)-m\left(t_{i}\right)\right) h_{t_{i}}(x)-d_{t_{j}}\left(t_{j}\right)\left(m(x)-m\left(t_{j}\right)\right) h_{t_{j}}(x)=w(x) \sum_{s \in T} c_{s}^{t_{i}, t_{j}} h_{s}(x)$. 
giving rise to syzygies $\Sigma_{t_{i}, t_{j}} \in S(H)$ of $H:=\left(h_{t}: t \in T\right)$, associated to the pairs $t_{i}, t_{j} \in T$ whose components

$$
\begin{aligned}
& \sigma_{t_{i}}^{t_{i}, t_{j}}(x)=w(x) c_{t_{i}}^{t_{i}, t_{j}}-d_{t_{i}}\left(t_{i}\right)\left(m(x)-m\left(t_{i}\right)\right), \\
& \sigma_{t_{j}, t_{j}}(x)=w(x) c_{t_{j}}^{t_{i}, t_{j}}+d_{t_{j}}\left(t_{j}\right)\left(m(x)-m\left(t_{j}\right)\right), \\
& \sigma_{s}^{t_{i}, t_{j}}(x)=w(x) c_{s}^{t_{i}, t_{j}}, \quad s \in T \backslash\left\{t_{i}, t_{j}\right\},
\end{aligned}
$$

are polynomials in $\Pi_{1}$.

Note that the syzygies $\Sigma_{t_{i}, t_{j}}, t_{i}, t_{j} \in T$, satisfy

$$
\Sigma_{t_{i}, t_{j}}+\Sigma_{t_{j}, t_{l}}=\Sigma_{t_{i}, t_{l}}, \quad t_{i}, t_{j}, t_{l} \in T .
$$

In particular, $\Sigma_{t_{i}, t_{i}}=0$ and $\Sigma_{t_{i}, t_{j}}=-\Sigma_{t_{j}, t_{i}}$.

We now focus on the syzygies

$$
\Sigma_{t_{0}, t_{i}}, \quad i=1, \ldots, n+1 .
$$

and define, in accordance with $[11,12]$,

$$
\Sigma(x)=\left(\sigma_{t_{j}}^{t_{0}, t_{i}}(x)\right)_{i=1, \ldots, n+1, j=0, \ldots, n+1} \in \Pi_{1}^{(n+1) \times(n+2)} .
$$

as the polynomial matrix whose rows are the components of these syzygies. We observe that if $x \in W$, then $w(x)=0$ and the $(n+1) \times(n+1)$ submatrix of $\Sigma(x)$ formed with the $n+1$ last columns simplifies to a diagonal matrix, whose diagonal entries are

$$
\sigma_{t_{i}}^{t_{0}, t_{i}}(x)=d_{t_{i}}\left(t_{i}\right)\left(m(x)-m\left(t_{i}\right)\right)
$$

By definition of $m$ and (2), we have that $\sigma_{t_{i}}^{t_{0}, t_{i}}(x) \neq 0$ for $x \in W \backslash\left\{t_{i}\right\}$, which implies that the rank of $\Sigma(x)$ over the field of rational functions is $n+1$, in other words, the syzygies are independent. Whenever we speak of the rank of a syzygy matrix it has to be understood in that sense.

The relations $\Sigma(x) H=0$ determine $H$ up to a polynomial factor. Let $\Sigma_{j}(x)$ be the submatrix obtained by removing the column corresponding to the index $j$, then $\operatorname{det} \Sigma_{j}(x) \in \Pi_{n+1}$ and there exists $a \in \mathbb{R}$ such that

$$
h_{t_{j}}(x)=(-1)^{j} a \operatorname{det} \Sigma_{j}(x), \quad j=0, \ldots, n+1 .
$$

In order to determine $a$ we restrict $x$ to the points on the line $W$, so that $w(x)=0$, and obtain, for $x \in W$, that

$$
\frac{d_{t_{0}}(x)}{d_{t_{0}}\left(t_{0}\right)}=h_{t_{0}}(x)=a \prod_{i=1}^{n+1} d_{t_{i}}\left(t_{i}\right)\left(m(x)-m\left(t_{i}\right)\right)=a d_{t_{0}}(x) \prod_{i=1}^{n+1} d_{t_{i}}\left(t_{i}\right),
$$

from which we deduce that

$$
a=\left(\prod_{t \in T} d_{t}(t)\right)^{-1}
$$


and

$$
h_{t_{j}}(x)=(-1)^{j}\left(\prod_{t \in T} d_{t}(t)\right)^{-1} \operatorname{det} \Sigma_{j}(x) .
$$

Thus we have shown that the syzygy matrix $\Sigma(x)$ has nonzero minors det $\Sigma_{j}$ for all $j=0, \ldots, n+1$. Furthermore, the minors are polynomials of exact degree $n+1$ in $x$.

Let us summarize the results obtained so far for further reference.

Theorem 4 Let $Y_{n}$ be a n-poised set, then there exists for any H-basis $\left(h_{0}, \ldots, h_{n+1}\right)$ of $I\left(Y_{n}\right)$ a syzygy matrix $\Sigma(x) \in \Pi_{1}^{(n+1) \times(n+2)}$ of rank $n+1$. The syzygy matrix determines the H-basis up to a constant factor $a \neq 0$

$$
h_{j}(x)=(-1)^{j} a \operatorname{det} \Sigma_{j}(x), \quad j=0, \ldots, n+1,
$$

where $\Sigma_{j}(x)$ denotes the submatrix obtained from $\Sigma(x)$ by removing the column corresponding to the index $j$.

Proof We have constructed $\Sigma(x)$ for a particular H-basis. Since all H-bases with $n+2$ elements of $I\left(Y_{n}\right)$ are bases of $\Pi_{n+1} \cap I\left(Y_{n}\right)$, they are related by a nonsingular matrix $B \in \mathbb{R}^{(n+2) \times(n+2)}$. Multiplying $\Sigma(x)$ from the right with $B$, we obtain the syzygy matrix $\Sigma(x) B \in \Pi_{1}^{(n+1) \times(n+2)}$ of rank $n+1$ for an arbitrary $\mathrm{H}$-basis $B^{-1} H$.

Theorem 5 Let $Y_{n}$ be a n-poised set. If $\Sigma(x), \Sigma^{\prime}(x) \in \Pi_{1}^{(n+1) \times(n+2)}$ are syzygy matrices of rank $n+1$ for two H-bases of $I\left(Y_{n}\right)$, then there exist nonsingular scalar matrices $A$ and $B$, such that

$$
\Sigma^{\prime}(x)=A \Sigma(x) B
$$

i.e., the linear syzygy matrix of rank $n+1$ for $I\left(Y_{n}\right)$ is unique up to equivalence.

Proof Let us denote by $S_{1}(H)=S(H) \cap \Pi_{1}^{n+2}$ the space of linear syzygies of a given H-basis of $I\left(Y_{n}\right)$. By Theorem 4, there exists a linear syzygy matrix $\Sigma(x)$ with respect to the particular H-basis

$$
H=\left(h_{j}(x):=E_{Y_{n}}\left[x_{1}^{n+1-j} x_{2}^{j}\right]: j=0, \ldots, n+1\right),
$$

where $E_{Y_{n}}[f]$ is the interpolation error to $f$ on $Y_{n}$ from $\Pi_{n}$. We will now show that any syzygy in $S_{1}(H)$ can be written as a linear combination of the rows of $\Sigma(x)$. Since

$$
x_{2} E_{Y_{n}}\left[x_{1}^{n+1-i} x_{2}^{i}\right](x)-x_{1} E_{Y_{n}}\left[x_{1}^{n-i} x_{2}^{i+1}\right](x)=\sum_{j=0}^{n+1} c_{i j} E_{Y_{n}}\left[x_{1}^{n+1-j} x_{2}^{j}\right](x),
$$

the corresponding syzygy matrix for $H$ can be written as

$$
\Sigma(x)=\left(\begin{array}{ccccc}
c_{00}-x_{2} & c_{01}+x_{1} & c_{02} & \ldots & c_{0, n+1} \\
c_{10} & c_{11}-x_{2} & c_{12}+x_{1} & \ldots & c_{1, n+1} \\
\vdots & \ddots & \ddots & \ddots & \vdots \\
c_{n 0} & \ldots & c_{n, n-1} & c_{n, n}-x_{2} & c_{n, n+1}+x_{1}
\end{array}\right)
$$


Now assume that $Z(x)$ is another syzygy in $S_{1}(H)$. We subtract a proper multiple of the first row of $\Sigma(x)$ from $Z(x)$ in such a way that the second component does not depend on $x_{1}$, then use the second row to eliminate $x_{1}$ from the third component, and so on. The resulting syzygy $Z(x)-b^{T} \Sigma(x)$, $b \in \mathbb{R}^{n+1}$ is of the form

$$
\left(\sigma_{0}\left(x_{1}, x_{2}\right), \sigma_{1}\left(x_{2}\right), \ldots, \sigma_{n+1}\left(x_{2}\right)\right) .
$$

But then

$0=\sum_{j=0}^{n+1} \sigma_{j}(x) h_{j}(x)=\sigma_{0}\left(x_{1}, x_{2}\right) E_{Y_{n}}\left[x_{1}^{n+1}\right](x)+\sum_{j=1}^{n+1} \sigma_{j}\left(x_{2}\right) E_{Y_{n}}\left[x_{1}^{n+1-j} x_{2}^{j}\right](x)$

implies that $\sigma_{0}=0$ because it is the coefficient of the only appearance of the power $x_{1}^{n+1}$. We can inductively apply the same reasoning to the powers $x_{1}^{n+1-j} x_{2}^{j}$ to conclude that $\sigma_{j}=0, j=1, \ldots, n$. Therefore $Z(x)=a^{T} \Sigma(x)$, that is, $Z(x)$ is a linear combination of the rows of $\Sigma(x)$, hence $\operatorname{dim} S_{1}(H)=$ $n+1$.

So we know that two linear syzygy matrices of rank $n+1$ for the same $\mathrm{H}$ basis are related by left multiplication by a nonsingular matrix. On the other hand, the proof of Theorem 4 tells us that changes of the H-bases correspond to right multiplication by a nonsingular matrix.

\section{Maximal lines}

Maximal lines, or, more generally, maximal hyperplanes, as introduced in [4], are at the heart of the Gasca-Maeztu conjecture. A $\Pi_{n}$-poised set $Y_{n}$ is said to contain a maximal line if there exists a line $W$ such that $\#\left(Y_{n} \cap W\right)=n+1$. As pointed out first by $\mathrm{H}$. Schenck, the following result that closely connects maximal lines to the syzygy matrix $\Sigma(x)$, can be seen as a special case of the Hilbert-Burch theorem, cf. [11]. We restate this fact here and give a more direct, affine and elementary proof of it.

Proposition 1 Suppose that $Y_{n}$ is a $\Pi_{n}$-poised set and there exist linearly independent $h_{0}, \ldots, h_{n+1} \in \Pi_{n+1} \cap I\left(Y_{n}\right)$ with a syzygy matrix $\Sigma \in \Pi_{1}^{(n+1) \times(n+2)}$ of rank $n+1$ with one column of the form $w(x) v, v \in \mathbb{R}^{n+1}$, for some nonconstant $w \in \Pi_{1}$. Then $W=V(w)$ is a maximal line for $Y_{n}$, i.e., \# $\left(W \cap Y_{n}\right)=$ $n+1$.

Proof Since the polynomials $h_{0}, \ldots, h_{n+1}$ generate $I\left(Y_{n}\right)$ and $\operatorname{dim}\left(\Pi_{n+1} \cap\right.$ $\left.I\left(Y_{n}\right)\right)=n+2$, it follows from Lemma 1 that these polynomials form an $H$-basis of $I\left(Y_{n}\right)$. After renumbering the ideal basis, we can assume that the last column of $\Sigma(x)$ is of the form $w(x) v$ for some vector $v$. By Theorem 4, $h_{j}=a(-1)^{j} \operatorname{det} \Sigma_{j} \in \Pi_{n+1}, j=0, \ldots, n+1$, up to a nonzero constant factor $a$. It follows that $h_{j}(x):=w(x) g_{j}(x), g_{j} \in \Pi_{n}, j=0, \ldots, n$, all belong to $I\left(Y_{n}\right)$ and that $g_{j} \in I\left(Y_{n} \backslash W\right), j=0, \ldots, n$, are $n+1$ linearly independent polynomials of degree $n$ in $I\left(Y_{n} \backslash W\right)$. If $q \in I\left(Y_{n} \backslash W\right) \cap \Pi_{n-1}$, then 
$q w \in \Pi_{n}$ vanishes on $Y_{n}$. Since $Y_{n}$ is $\Pi_{n}$-poised, it follows that $q=0$. So we have $I\left(Y_{n} \backslash W\right) \cap \Pi_{n-1}=0$. By Lemma $1,\left(g_{j}: j=0, \ldots, n\right)$ is an $H$-basis of $I\left(Y_{n} \backslash W\right)$. Since $\Pi=\Pi_{n-1} \oplus I\left(Y_{n} \backslash W\right)$, we deduce that $Y_{n} \backslash W$ is $\Pi_{n-1}$-poised and

$$
\#\left(Y_{n} \cap W\right)=\operatorname{dim} \Pi_{n}-\#\left(Y_{n} \backslash W\right)=\operatorname{dim} \Pi_{n}-\operatorname{dim} \Pi_{n-1}=n+1
$$

which means that $W$ is indeed a maximal line.

Since one can apply row transformations to the syzygy matrix to obtain another syzygy matrix for the same basis, the following statement is equivalent to Proposition 1.

Corollary 2 Suppose that $Y_{n}$ is a $\Pi_{n}$-poised set and there exist linearly independent $h_{0}, \ldots, h_{n+1} \in \Pi_{n+1} \cap I\left(Y_{n}\right)$ with a syzygy matrix $\Sigma \in \Pi_{1}^{(n+1) \times(n+2)}$ of rank $n+1$ whose $j$-th column is of the form $w(x) e_{i}$ for some linear function $w$ and some $i \in\{1, \ldots, n+1\}$ and $j \in\{0, \ldots, n+1\}$. Then $W$ is a maximal line for $Y_{n}$.

The results from the preceding section also allow us to give a converse statement of Proposition 1 that says that any maximal line can be found in a proper syzygy matrix.

Proposition 2 If a $\Pi_{n}$-poised set $Y_{n} \subset \mathbb{R}^{2}$ contains a maximal line $W$, then there exists a basis $h_{0}, \ldots, h_{n+1}$ of $\Pi_{n+1} \cap I\left(Y_{n}\right)$ with a syzygy matrix $\Sigma \in$ $\Pi_{1}^{(n+1) \times(n+2)}$ of rank $n+1$ whose last column is of the form $w(x) e_{n+1}$.

Proof Let $Y_{n-1}$ be any $(n-1)$-poised set in $\mathbb{R}^{2}$ and $W$ be a line with $W \cap Y_{n-1}=$ $\emptyset$. Choose $n+1$ points $t_{0}, \ldots, t_{n}$ on $W$ and let $Y_{n}$ be the union of $Y_{n-1}$ and these $n+1$ points. Now set, with the notation from the preceding section,

$g_{j}(x):=w(x) h_{t_{j}}(x), \quad j=0, \ldots, n, \quad g_{n+1}(x):=-\left(m(x)-m\left(t_{0}\right)\right) h_{t_{0}}(x)$.

These polynomials are linearly independent. Indeed, evaluating

$$
\sum_{j=0}^{n+1} c_{j} g_{j}(x)=0
$$

along $W$ yields $c_{n+1}=0$ and then

$$
0=w(x) \sum_{j=0}^{n} c_{j} h_{t_{j}}(x),
$$

which implies that $c_{0}=\cdots=c_{n}=0$. Since, in addition, $g_{j}\left(Y_{n}\right)=0$, it follows from Lemma 1 that $g_{0}, \ldots, g_{n+1}$ are an H-basis of $I\left(Y_{n}\right)$. The syzygy matrix for this basis takes the form

$$
\left(\begin{array}{ccccc}
\sigma_{t_{0}}^{t_{0}, t_{1}}(x) & \sigma_{t_{1}}^{t_{0}, t_{1}}(x) & \ldots & \sigma_{t_{n}}^{t_{0}, t_{1}}(x) & 0 \\
\vdots & \vdots & \ddots & \vdots & \vdots \\
\sigma_{t_{0}}^{t_{0}, t_{n}}(x) & \sigma_{t_{1}}^{t_{0}, t_{n}}(x) & \ldots & \sigma_{t_{n}}^{t_{0}, t_{n}}(x) & 0 \\
m(x)-m\left(t_{0}\right) & 0 & \ldots & 0 & w(x)
\end{array}\right),
$$


with $\sigma_{t_{i}}^{t_{0}, t_{i}}$ given by (4). The upper left part of this matrix is the syzygy matrix for $Y_{n} \backslash W$. The last column now consists of the linear polynomial $w$ marking the maximal line.

Combining Proposition 1, Proposition 2 and Theorem 5, we can now even give a characterization of maximal lines in terms of syzygies.

Theorem 6 A $\Pi_{n}$-poised set $Y_{n} \subset \mathbb{R}^{2}$ contains a maximal line if and only if there exists a basis $h_{0}, \ldots, h_{n+1}$ of $\Pi_{n+1} \cap I\left(Y_{n}\right)$ with a syzygy matrix $\Sigma \in$ $\Pi_{1}^{(n+1) \times(n+2)}$ of rank $n+1$ whose $j$-th column is of the form $w(x) e_{i}$ for some polynomial $w$ of degree 1 and some $i \in\{1, \ldots, n+1\}$ and $j \in\{0, \ldots, n+1\}$. The polynomial $w$ determines the maximal line $W=V(w)$.

\section{Syzygy matrices of $G C_{n}$ sets}

We recall that a $G C_{n}$ set, introduced by Chung and Yao [10], is a $\Pi_{n}$-poised set whose Lagrange fundamental polynomials are products of linear factors. Gasca and Maeztu conjectured in [13] that any $G C_{n}$ set contains a maximal line. In this section we consider some special $G C_{n}$ sets and their syzygy matrices, namely the two most important and best investigated $[2,8]$ examples of $G C_{n}$ sets: natural lattices and (generalized) principal lattices. We start with the natural lattice $Y_{n}$ corresponding to the intersections of $n+2$ lines $W_{0}, \ldots, W_{n+1}$ in general position, such that $W_{i} \cap W_{j}, 0 \leq i<j \leq n+1$, form a set of $\left(\begin{array}{c}n+2 \\ 2\end{array}\right)$ points $x_{i j}$ in $\mathbb{R}^{2}$. The Lagrange fundamental polynomial $\ell_{i j}$ corresponding to $x_{i j}$ is then of the form

$$
\ell_{i j}(x)=\prod_{r \in\{0, \ldots, n+1\} \backslash\{i, j\}} \frac{w_{r}(x)}{w_{r}\left(x_{i j}\right)} .
$$

Then we can construct an H-basis of $I\left(Y_{n}\right)$ basis using Theorem 2. Let $W_{n+2}$ be a line intersecting all previous lines at $x_{i, n+2}$, then

$$
\ell_{i, n+2}(x)=\prod_{r \in\{0, \ldots, n+1\} \backslash\{i\}} \frac{w_{r}(x)}{w_{r}\left(x_{i, n+2}\right)} .
$$

So,

$h_{i}(x)=\ell_{i, n+2}(x) \prod_{r \neq i} w_{r}\left(x_{i, n+2}\right)=\prod_{r \in\{0, \ldots, n+1\} \backslash\{i\}} w_{r}(x), \quad i=0, \ldots, n+1$,

form an H-basis of $I\left(Y_{n}\right)$. For this H-basis we obtain the following syzygy matrix:

$$
\Sigma(x)=\left(\begin{array}{ccccc}
w_{0}(x) & -w_{1}(x) & 0 & \cdots & 0 \\
w_{0}(x) & 0 & -w_{2}(x) & \ddots & \vdots \\
\vdots & \vdots & \ddots & \ddots & 0 \\
w_{0}(x) & 0 & \cdots & 0 & -w_{n}(x)
\end{array}\right)
$$


Corollary 2 proves that $W_{j}, j=0 \ldots, n+2$, are maximal lines.

Now let us consider the case of a generalized principal lattice, introduced in $[6,7]$ and further analyzed in [9]. In this case, we have $3 n$ lines $W_{i, j}, i=$ $0, \ldots, n, j=0,1,2$, where

$$
W_{\beta_{0}, 0} \cap W_{\beta_{1}, 1} \cap W_{\beta_{2}, 2}=\left\{x_{\beta}\right\}, \quad|\beta|=n .
$$

are requested to be distinct. Then $Y_{n}=\left\{x_{\beta}:|\beta|=n\right\}$ is an $\Pi_{n}$-poised set and $G C_{n}$ because the corresponding Lagrange polynomials are of the form

$$
\ell_{\beta}(x)=\prod_{\gamma_{0}<\beta_{0}} \frac{w_{\gamma_{0}, 0}(x)}{w_{\gamma_{0}, 0}\left(x_{\beta}\right)} \prod_{\gamma_{1}<\beta_{1}} \frac{w_{\gamma_{1}, 1}(x)}{w_{\gamma_{1}, 1}\left(x_{\beta}\right)} \prod_{\gamma_{2}<\beta_{2}} \frac{w_{\gamma_{2}, 2}(x)}{w_{\gamma_{2}, 2}\left(x_{\beta}\right)} .
$$

One H-basis of $I\left(Y_{n}\right)$ is

$$
h_{j}(x)=\prod_{\gamma_{1}<j} w_{\gamma_{1}, 1}(x) \prod_{\gamma_{2}<n+1-j} w_{\gamma_{2}, 2}(x), \quad j=0, \ldots, n+1,
$$

whose syzygy matrix is of the form

$$
\Sigma(x)=\left(\begin{array}{ccccc}
w_{0,1}(x) & -w_{n, 2}(x) & 0 & \ldots & 0 \\
0 & w_{1,1}(x) & -w_{n-1,2}(x) & \ddots & \vdots \\
\vdots & \ddots & \ddots & \ddots & 0 \\
0 & \ldots & 0 & w_{n, 1}(x) & -w_{0,2}(x)
\end{array}\right) .
$$

Thus $W_{0,1}$ and $W_{0,2}$ turn out to be maximal lines by inspecting first and last column of the syzygy matrix. Since we left out any factor with $w_{j, 0}$ in the $\mathrm{H}_{-}$ basis (5), the line $W_{0,0}$ is not detected by the syzygy matrix. However, there must exist a basis transform that maps the polynomials from (5) to

$$
h_{j}^{\prime}(x)=\prod_{\gamma_{0}<j} w_{\gamma_{0}, 0}(x) \prod_{\gamma_{2}<n+1-j} w_{\gamma_{2}, 2}(x), \quad j=0, \ldots, n+1,
$$

which implies that a column transform of $\Sigma(x)$ then gives a column consisting only of a multiple of $w_{0,0}$.

The particular structure of the fundamental polynomials of $G C_{n}$ sets, namely the rare property that they can be factored into linear polynomials, and the preceding examples suggest the following construction of a factorizable H-basis for $I\left(Y_{n}\right)$ which is originally due to Schenck in an unpublished manuscript and for which we can now give a more direct and elementary exposition. To this end, we choose a point $z \in \mathbb{R}^{2}$ such that $\ell_{y, Y_{n}}(z) \neq 0, y \in Y_{n}$, and define the sets

$$
\mathcal{L}(y):=\left\{m \in \Pi_{1}: m \mid \ell_{y, Y_{n}}, m(z)=1\right\}, \quad y \in Y_{n}, \quad \mathcal{L}:=\bigcup_{y \in Y_{n}} \mathcal{L}(y) .
$$

The purpose of the point $z$ is only to uniquely normalize the polynomials in $\mathcal{L}$. Moreover, let $Y_{n-1}$ be any $\Pi_{n-1}$-poised subset of $Y_{n}$ and set $T:=Y_{n} \backslash Y_{n-1}$. 
Proposition 3 The set

$$
\bigcup_{t \in T}\left\{m \ell_{t, Y_{n}}: m \in \mathcal{L}, m(t)=0\right\}
$$

contains an H-basis for $I\left(Y_{n}\right)$ whose elements are products of first degree polynomials.

Proof Since there always exist at least two nonparallel lines belonging to $\mathcal{L}$ that vanish on $t$, we find that

$$
\operatorname{span}\left\{m \ell_{t, Y_{n}}: m \in \mathcal{L}, m(t)=0\right\}=\operatorname{span}\left\{x_{1}-t_{1}, x_{2}-t_{2}\right\} \ell_{t, Y_{n}} .
$$

Since, on the other hand,

$$
\operatorname{span}\left\{\ell_{t, Y_{n}}: t \in T\right\}=\operatorname{span}\left\{E_{Y_{n-1}}\left[(\cdot)^{\alpha}\right]:|\alpha|=n\right\},
$$

it follows that the space

$$
\sum_{t \in T} \operatorname{span}\left\{m \ell_{t, Y_{n}}: m \in \mathcal{L}, m(t)=0\right\} \subset I\left(Y_{n}\right)
$$

contains all polynomials of the form $E_{Y_{n}}\left[x^{\alpha}\right],|\alpha|=n+1$. Indeed, write, for some $\alpha$ with $|\alpha|=n$

$$
E_{Y_{n-1}}\left[x^{\alpha}\right]=\sum_{t \in T} c_{t} \ell_{t, Y_{n}}(x)
$$

and use $\epsilon_{1}:=(1,0)$ and $\epsilon_{2}:=(0,1)$ for the unit multiindices in $\mathbb{N}_{0}^{2}$. It then follows for $j=1,2$, that

$$
x_{j} E_{Y_{n-1}}\left[x^{\alpha}\right]-\sum_{t \in T} c_{t} t_{j} \ell_{t, Y_{n}}(x)=\sum_{t \in T} c_{t}\left(x_{j}-t_{j}\right) \ell_{t, Y_{n}}(x),
$$

is a polynomial of degree $n+1$ which vanishes on $Y_{n}$. This polynomial coincides with $E_{Y_{n}}\left[x^{\alpha+\epsilon_{j}}\right]$ because $x^{\alpha+\epsilon_{j}}-x_{j} E_{Y_{n-1}}\left[x^{\alpha}\right] \in \Pi_{n}$. So, we have

$$
E_{Y_{n}}\left[x^{\alpha+\epsilon_{j}}\right]=\sum_{t \in T} c_{t}\left(x_{j}-t_{j}\right) \ell_{t, Y_{n}}(x)
$$

and therefore the set from (7) spans $\Pi_{n+1} \cap I\left(Y_{n}\right)$. By Corollary 1 the set is an $\mathrm{H}$-basis as claimed. Since all candidates are factorizable by construction, so is the resulting $\mathrm{H}$-basis.

Remark 1 Proposition 3 can also be found in [12] where it is in turn attributed as implicitly given already in [18].

Acknowledgements We a very grateful to Hal Schenck for the discussions on a draft of his paper that helped us to write this paper and to Carl de Boor for a lot of valuable remarks and comments on the first draft that greatly improved the presentation. 


\section{References}

1. Berzolari, L.: Sulla determinazione di una curva o di una superficie algebrica e su algune questioni di postulazione. Lomb. Ist. Rend. 47, 556-564 (1914)

2. de Boor, C.: The error in polynomial tensor-product, and in Chung-Yao, interpolation. In: A. Le Méhauté, C. Rabut, L.L. Schumaker (eds.) Surface Fitting and Multiresolution Methods, pp. 35-50. Vanderbilt University Press (1997)

3. de Boor, C.: Ideal interpolation. In: C.K. Chui, M. Neamtu, L.L. Schumaker (eds.) Approximation Theory XI, Gaitlinburg 2004, pp. 59-91. Nashboro Press (2005)

4. de Boor, C.: Multivariate polynomial interpolation: conjectures concerning GC-sets. Numer. Algor. 45, 113-125 (2007)

5. Carnicer, J., Gasca, M.: Classification of bivariate GC configurations for interpolation. Advances Comput. Math. 20, 5-16 (2004)

6. Carnicer, J., Gasca, M.: Generation of lattices of points for bivariate interpolation. Numer. Algo. 39, 69-79 (2005)

7. Carnicer, J., Gasca, M.: Interpolation on lattices generated by cubic pencils. Advances Comput. Math. 24, 113-130 (2006)

8. Carnicer, J., Gasca, M., Sauer, T.: Aitken-Neville sets, principal lattices and divided differences. J. Approx. Theor. 156, 154-172 (2009)

9. Carnicer, J., Godés, C.: Geometric characterization and generalized principal lattices. J. Approx. Theory 143, 2-14 (2006)

10. Chung, K.C., Yao, T.H.: On lattices admitting unique Lagrange interpolation. SIAM J. Num. Anal. 14, 735-743 (1977)

11. Eisenbud, D.: The Geometry of Syzygies. A Second Course in Algebraic Geometry and Commutative Algebra. Springer (2005)

12. Fieldsteel, N., Schenck, H.: Polynomial interpolation in higher dimension: From simplicial complexes to $G C$ sets (2016). Preprint

13. Gasca, M., Maeztu, J.I.: On Lagrange and Hermite interpolation in $\mathbb{R}^{k}$. Numer. Math. 39, 1-14 (1982)

14. Gasca, M., Sauer, T.: On the history of multivariate polynomial interpolation. J. Comput. Appl. Math. 122, 23-35 (2000)

15. Hakopian, H., Jetter, K., Zimmermann, G.: The Gasca-Maeztu conjecture for $n=5$. Numer. Math. 127, 685-713 (2014)

16. Lorentz, G.G.: Approximation of functions. Chelsea Publishing Company (1966)

17. Radon, J.: Zur mechanischen Kubatur. Monatshefte der Math. Physik 52, 286-300 (1948)

18. Sauer, T., Xu, Y.: On multivariate Lagrange interpolation. Math. Comp. 64, 1147-1170 (1995)

19. Steffensen, I.F.: Interpolation. Chelsea Pub., New York (1927) 